Research Paper

\title{
The comparison of EGFR-TKI failure modes and subsequent management between exon 19 deletion and exon 21 L858R mutation in advanced non-small-cell lung
} cancer

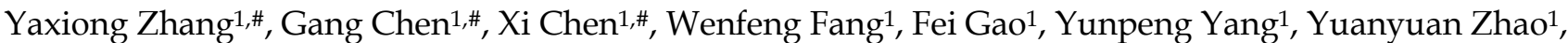 \\ Yuxiang Ma1, Shaodong Hong1, Zhonghan Zhang', Siyu Miao², Manli Wu², Xiaodan Huang2, Youli Luo ${ }^{3}$, \\ Cong Zhou ${ }^{3}$, Run Gong3, Yan Huang1, Likun Chen'1, Ningning Zhou1, Hongyun Zhao1, Li Zhang1, \\ 1. Sun Yat-sen University Cancer Center, State Key Laboratory of Oncology in South China, Collaborative Innovation Center for Cancer Medicine, \\ Guangzhou, China; \\ 2. Zhongshan School of Medicine, Sun Yat-sen University, Guangzhou, China; \\ 3. The Fifth Affiliated Hospital of Sun Yat-Sen University, Zhu Hai, China; \\ \#These authors contributed equally to this work. \\ $\triangle$ Corresponding author: Li Zhang, Department of Medical Oncology, Sun Yat-sen University Cancer Center, 651 Dongfeng Road East, Guangzhou, \\ Guangdong 510060, P.R. China. Tel: +86-20-87343458. Fax: +86-20-87343535. Email: zhangli6@mail.sysu.edu.cn. \\ (C) Ivyspring International Publisher. This is an open access article distributed under the terms of the Creative Commons Attribution (CC BY-NC) license \\ (https://creativecommons.org/licenses/by-nc/4.0/). See http://ivyspring.com/terms for full terms and conditions.
}

Received: 2017.03.01; Accepted: 2017.05.10; Published: 2017.07.02

\begin{abstract}
Background: Advanced non-small-cell lung cancer (NSCLC) with epidermal growth factor receptor (EGFR) exon 19 deletion (19 Del) and exon 21 L858R mutation (L858R) might be distinct diseases. Therefore, it is necessary to take EGFR mutation subgroups into consideration for making choices of subsequent treatment after tyrosine kinase inhibitors (TKIs) failure.

Patients and methods: 174 patients who developed to EGFR-TKI failure were categorized into three cohorts of dramatic progression, gradual progression and local progression. Chi-square was used to compare the distribution of failure modes between 19 Del and L858R. Kaplan-Meier method and Cox Regression were performed for analyses of survival in different subsequent treatments.

Results: The distribution of EGFR-TKI failure modes showed no significant difference between 19 Del and L858R. Patients in gradual progression had a longer progression-free survival (PFS) and overall survival (OS) compared with other failure modes in whole population, 19 Del cohort and L858R cohort. 19 Del patients with dramatic progression would obtain survival benefit from chemotherapy, while those with gradual progression got no survival benefit neither from chemotherapy nor previous TKI continuation. However, patients with dramatic or gradual progression would benefit from previous TKI continuation in L858R cohort.

Conclusion: For advanced EGFR-positive NSCLC patients with acquired resistance to EGFR-TKI, subsequent treatment should be personalized according to EGFR-TKI failure modes \& EGFR mutation subtypes.
\end{abstract}

Key words: NSCLC, EGFR-TKI, 19 Del, L858R, clinical failure mode, subsequent treatment

\section{Introduction}

Epidermal growth factor receptor tyrosine kinase inhibitors (EGFR-TKIs) such as gefitinib, erlotinib or afatinib have been recognized as a standard first-line therapy for non-small-cell lung cancer (NSCLC) patients who harboring EGFR mutations $[1,2,3,4]$. However, the therapeutic efficacy and survival benefits of EGFR-TKI in EGFR-positive patients were different between 
patients with exon 19 Deletion (19 Del) and exon 21 L858R mutation (L858R) [5]. Afatinib improved overall survival (OS) of 19 Del patients, instead of those with L858R[6].A meta-analysis also showed 19 Del might be associated with longer progression-free survival (PFS) compared to L858R after first-line EGFR-TKIs [7]. Moreover, previous study showed that the baseline clinical characteristics were different between 19 Del and L858R. Patients with 19 Del were more likely to be young and had lymphatic metastasis than those with L858R [8]. All indicated that these two kinds of common EGFR mutants were different diseases and might require different management.

Unfortunately, EGFR-positive patients who benefited from TKIs finally developed to acquired resistance [9].On the one hand, secondary EGFR exon 20 T790M mutation (T790M), c-Met amplification and tumor type transformation were thought to be molecular modes of EGFR-TKI failure. 3rd-generation TKI, previous TKI plus c-Met inhibitor and chemotherapy were recommended subsequent treatment, respectively $[10,11,12]$. On the other hand, researchers categorized EGFR-TKI failure into three clinical modes (dramatic progression, gradual progression and local progression) by different duration of disease control, evolution of tumor burden, and clinical symptoms. Different subsequent measures were suggested for patients with different clinical EGFR-TKI failure modes. For dramatic progression, patients should turn to chemotherapy immediately. For gradual progression, patients might continue previous TKI treatment. For local progression, continuation of previous TKI plus local intervention was recommended [13].

Considering that $19 \mathrm{Del}$ and L858R are different disease, however, it is still unknown whether the clinical modes of EGFR-TKI failure are distinct between 19 Del and L858R. Moreover, whether the subsequent management after TKI failure should be different between 19 Del and L858R is worth exploring. Therefore, we conduct this study to compare the clinical modes of EGFR-TKI failure and subsequent management between $19 \mathrm{Del}$ and L858R in advanced NSCLC patients.

\section{Methods}

\section{Study population}

1271 pathologically confirmed NSCLC patients who ordered detection of EGFR gene mutation tests with either 19 Del or L858R at the Cancer Center of Sun Yat-sen University (Guangzhou, China) from October 2008 to July 2013 were enrolled in this study. 485of them didn't have adequate clinical data such as baseline characteristic, treatment history and survival data for analyses thus were excluded. Till to the last follow-up time (June, 2016) there were 174 advanced EGFR-positive patients using 1st-generation TKIs developed to progression diseases. Figure $\mathbf{1}$ summarizes the flow chart of the included cases. The objective tumor response was determined by professional physicians according to RECIST v1.1 (Response Evaluation Criteria in Solid Tumors, version 1.1) [14]. The clinicopathological features of the patients including gender, age, smoking status, TNM stage, histologic type and treatment records were collected from the SYSUCC HIS (Sun Yat-Sen University Cancer Center Hospital Information System) by well-trained physicians. All the patients had provided written informed consent before treatment.

\section{Categorization of EGFR-TKI failure clinical modes}

The clinical modes of EGFR-TKI failure were evaluated and validated by 3 independent physicians according to the study of Jin-Ji Yang [13]. The criteria of each mode were introduced briefly as follows: (1) dramatic progression, 1) disease control $\geq 3$ months with EGFR-TKI treatment; 2) compared with previous assessment, rapid increment of tumor burden; 3) symptom scored 2. (2) gradual progression, 1) Disease control $\geq 6$ months with EGFR-TKI treatment; 2) Compared with the previous assessment, minor increment of tumor burden; 3) symptom scored $\leq 1$. (3) local progression, 1) disease control $\geq 3$ months with EGFR-TKI treatment; 2) Solitary extracranial progression or intracranial progression; 3) symptom scored $\leq 1$. Symptom scores 0,1 and 2 was quantified as asymptomatic status, stability of pre-existing item, and deterioration of any pre-existing item or new item, respectively [15].

\section{EGFR mutation detection}

EGFR mutations were detected using PCR-based direct sequencing of exons 18-21. The method is briefly introduced as follows. First, genomic DNA was extracted from tumors embedded in paraffin blocks or fresh frozen tumors. Then, use Hot Star Taq DNA polymerase (Qiagen Inc, Valencia, CA) to complete PCR amplification with a forward primer (50-GGATCGGCCTCTTCATGC-30) and a reverse primer (50-TAAAATTGATTCCAATGCCATCC-30). Sequencing was performed by ABI PRISM 3100 Genetic Analyzer (Applied Biosystems, Foster City, CA) using Applied Biosystems PRISM dye terminator cycle sequencing method (Perkin-Elmer Corp., Foster City, CA) directly on PCR products. Any in-frame 19 Del or point mutations in exon 21, which confer sensitivity to EGFR-TKIs therapy, were considered EGFR mutant. 


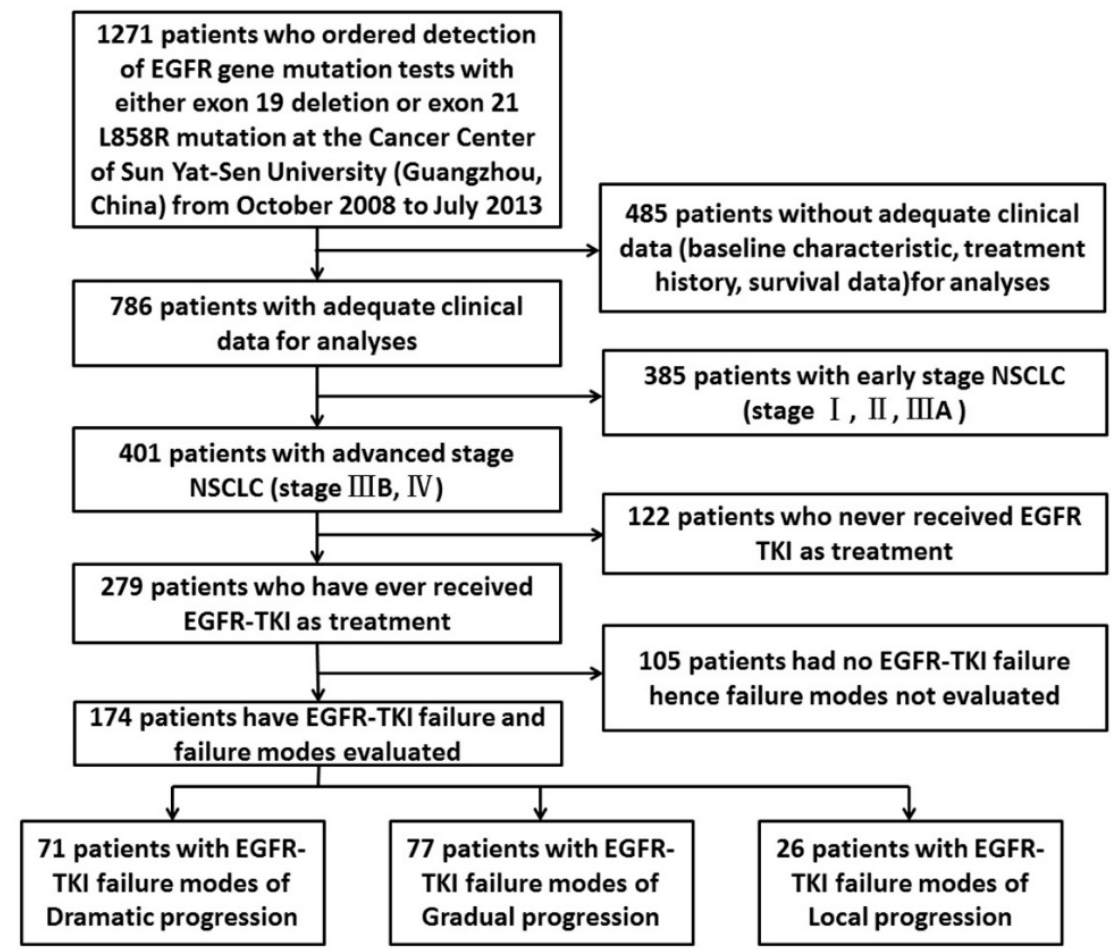

Figure 1. Flow chart of the enrollment. Abbreviations: EGFR, epidermal growth factor receptor; NSCLC, non-small-cell lung cancer; TKI, tyrosine kinase inhibitor.

\section{Therapeutic outcomes and statistical analyses}

PFS and OS were adopted as main therapeutic outcomes. PFS was defined as the time from beginning of 1st-generation EGFR-TKI treatment to the first radiographic proof of progressive disease (PD) or death from any cause. OS was calculated from the time when patients were pathological diagnosed to the last follow up or death from any cause. The median follow-up time of OS for the whole 174 patients was 65.0 months. IBM SPSS Statistics 23.0 software was used for the statistical analysis. Chi-square was used to compare qualitative data. Kaplan-Meier method with log-rank test was used to estimate the survival curves. Cox Regression was performed for multivariate analyses of survival. The prognostic results were reported with median survival, hazard ratio (HR) and its 95\% confidence interval (CI). HR $<1$ implied a lower risk of progression or death for patients. All statistical tests were two-sided and P-value $<0.05$ was considered to be statistically significant.

\section{Results}

\section{Enrolled patients}

A total of 174 advanced EGFR-positive NSCLC patients who developed 1st-generation EGFR-TKI failure were enrolled in this study. Both 19Del and L858R had 87 patients in each group. The distribution of EGFR-TKI failure modes showed 71 cases with dramatic progression, 77 cases with gradual progression and 26 cases with local progression. Basic clinicopathological features of the patients were presented in Table 1. There was no difference in the distribution of all basic characteristics between 19Del and L858R.

\section{Comparison of EGFR-TKI failure modes between 19Del and 21 L858R}

Figure 2 showed the distributions of EGFR-TKI failure clinical modes were 34 cases (39.1\%)with dramatic progression, 41 cases $(47.1 \%)$ with gradual progression and 12 cases $(13.8 \%)$ with local progression among $19 \mathrm{Del}$, which were similar to L858R (dramatic progression, $\mathrm{N}=37$ (42.5\%); gradual progression, $\mathrm{N}=36$ (41.4\%); local progression, $\mathrm{N}=14$ $(16.1 \%))$. The distribution of EGFR-TKI failure modes between 19Del and L858R showed no significant difference $(\mathrm{P}$-value $=0.739)$.

\section{Survival in different EGFR-TKI failure modes}

We conducted the comparisons of both PFS and OS among the whole population stratified by three clinical TKI failure modes. Patients with dramatic progression (median PFS $(\mathrm{mPFS})=6.1 \mathrm{~m})$, gradual progression $(\mathrm{mPFS}=15.6 \mathrm{~m})$ and local progression $(\mathrm{mPFS}=8.5 \mathrm{~m})$ showed significantly different PFS $($ P-value $<0.001)$ (Figure 3A). PFS results were still significantly different in the cohort of both 19Del and L858R (Figure 3B and 3C). Moreover, the OS results showed similar significantly different among the 
whole patients with different clinical TKI failure modes (P-value $<0.001$ ). The mOSwere $16.4 \mathrm{~m}, 26.5 \mathrm{~m}$ and $21.5 \mathrm{~m}$ in patients with dramatic, gradual and local progression, respectively (Figure 3D), which were similar in the cohort of both 19Del and L858R (Figure 3E and 3F). Our study demonstrated that patients in gradual progression had a longer PFS and OS compared with the other two progression modes in whole population, 19 Del cohort and L858R cohort.

Table 1. Characteristic of Enrolled 174 Nonsmall-Cell Lung Cancer Patients with Either Exon 19 Deletions or Exon 21 L858R Mutations.

\begin{tabular}{|c|c|c|c|c|c|c|}
\hline \multirow[t]{2}{*}{ Parameter } & \multirow[t]{2}{*}{ Total } & \multicolumn{2}{|c|}{19 Del } & \multicolumn{2}{|c|}{ L858R } & \multirow[t]{2}{*}{ P-value } \\
\hline & & $n$ & $\%$ & $n$ & $\%$ & \\
\hline Gender & & & & & & 1.00 \\
\hline Male & 82 & 41 & 47.1 & 41 & 47.1 & \\
\hline Female & 92 & 46 & 52.9 & 46 & 52.9 & \\
\hline Age (years) & & & & & & 0.17 \\
\hline Median (range) & $55(28-80)$ & 54 & 28-80) & 57 & 38-78) & \\
\hline Smoking status & & & & & & 0.14 \\
\hline Never & 136 & 64 & 73.6 & 72 & 82.8 & \\
\hline Smoker & 38 & 23 & 26.4 & 15 & 17.2 & \\
\hline Drinking status & & & & & & 0.70 \\
\hline Never & 140 & 71 & 81.6 & 69 & 79.3 & \\
\hline Drinker & 34 & 16 & 18.4 & 18 & 20.7 & \\
\hline Stage & & & & & & 1.00 \\
\hline IIIB & 2 & 1 & 1.1 & 1 & 1.1 & \\
\hline IV & 172 & 86 & 98.9 & 86 & 98.9 & \\
\hline Histology & & & & & & 1.00 \\
\hline $\mathrm{ADC}$ & 170 & 85 & 97.7 & 85 & 97.7 & \\
\hline Non-ADC & 4 & 2 & 2.3 & 2 & 2.3 & \\
\hline Line of TKI & & & & & & 0.76 \\
\hline $1 \mathrm{st}$ & 72 & 35 & 40.2 & 37 & 42.5 & \\
\hline$\geq 2$ nd & 102 & 52 & 59.8 & 50 & 57.5 & \\
\hline TKI failure modes & & & & & & 0.74 \\
\hline Dramatic progression & 71 & 34 & 39.1 & 37 & 42.5 & \\
\hline Gradual progression & 76 & 41 & 47.1 & 36 & 41.4 & \\
\hline Local progression & 36 & 12 & 13.8 & 14 & 16.1 & \\
\hline Subsequent treatment & & & & & & 0.25 \\
\hline BSC & 34 & 12 & 13.8 & 22 & 25.3 & \\
\hline Chemo & 102 & 57 & 65.5 & 45 & 51.7 & \\
\hline Pre TKI & 29 & 15 & 17.2 & 14 & 16.1 & \\
\hline 3rd-generation TKI & 6 & 2 & 2.3 & 4 & 4.6 & \\
\hline c-Met inhibitor + Pre TKI & 3 & 1 & 1.1 & 2 & 2.3 & \\
\hline Total & 174 & 87 & 50.0 & 87 & 50.0 & \\
\hline
\end{tabular}

Abbreviations: 19 Del, exon 19 deletion; L858R, exon 21 L858R mutation; ADC, adenocarcinoma; EGFR, epidermal growth factor receptor; Pre TKI, previous tyrosine kinase inhibitor; BSC, best supportive care; Chemo, chemotherapy.

\section{Survival in different subsequent treatments}

There were mainly three types of subsequent treatments, namely previous TKI (Pre TKI, N=29, $16.7 \%$ ), chemotherapy (Chemo, $\mathrm{N}=102,58.6 \%$ ) and best supporting treatment (BSC, $\mathrm{N}=34,19.5 \%$ ) after TKI failure. Only 6 patients used 3rd-generation TKI and 3 patients used c-Met inhibitor, which were not included in following analyses (Table 1).

In gradual progression group $(\mathrm{N}=71)$, patients with previous TKI continuation demonstrated a significantly longer OS $(\mathrm{mOS}=37.5 \mathrm{~m})$ than those switching to chemotherapy $(\mathrm{mOS}=27.4 \mathrm{~m})$ or $\mathrm{BSC}$ $(\mathrm{mOS}=19.5 \mathrm{~m}) \quad(\mathrm{P}$-value $=0.006$, Figure $4 \mathrm{~A})$. We found similar results in L858R subgroup (Pre TKI, $\mathrm{mOS}=49.5 \mathrm{~m}$; Chemo, $\mathrm{mOS}=27.0 \mathrm{~m}$; $\mathrm{BSC}, \mathrm{mOS}=18.0 \mathrm{~m}$; $\mathrm{P}$-value $=0.042$; Figure 4C). However, the results were negative in 19Del subgroup (Pre TKI, $\mathrm{mOS}=28.5 \mathrm{~m}$; Chemo, $\quad \mathrm{mOS}=27.8 \mathrm{~m}$; $\quad \mathrm{BSC}, \quad \mathrm{mOS}=25.5 \mathrm{~m}$; $\mathrm{P}$-value $=0.302$; Figure 4B). The survival benefit of previous TKI continuation were only found in L858R patients (P-value $=0.041$, Figure 4D), which was consistent with the result of multivariate analysis (Table S1).

In dramatic progression group $(\mathrm{N}=69)$, subsequent treatments showed similar OS (Pre TKI, $\mathrm{mOS}=15.8 \mathrm{~m}$; Chemo, $\mathrm{mOS}=17.7 \mathrm{~m}$; $\mathrm{BSC}, \mathrm{mOS}=14.5 \mathrm{~m}$; $P$-value $=0.164 ;$ Figure 4E). However, in 19 Del subgroup, patients switching to chemotherapy had longer OS $(\mathrm{mOS}=22.5 \mathrm{~m})$ than those with TKI continuation $(\mathrm{mOS}=10.5 \mathrm{~m})$ or $\mathrm{BSC} \quad(\mathrm{mOS}=14.3 \mathrm{~m})$ ( $\mathrm{P}$-value $=0.005$, Figure 4F). On the contrary, in L858R subgroup, patients with previous TKI continuation demonstrated a numerical longer OS $(\mathrm{mOS}=33.0 \mathrm{~m})$ than those switching to chemotherapy $(\mathrm{mOS}=12.0 \mathrm{~m})$ or BSC $(\mathrm{mOS}=15.0 \mathrm{~m})(\mathrm{P}$-value $=0.260$, Figure $4 \mathrm{G})$. The merged results showed the survival benefits of switching to chemotherapy or previous TKI continuation were in 19 Del or L858R patients, respectively (P-value $=0.059$, Figure $4 \mathbf{H})$, which was similar to multivariate analysis(Table S2).

Only 25 patients were classified as local progression, so they were not included in subgroup analyses.

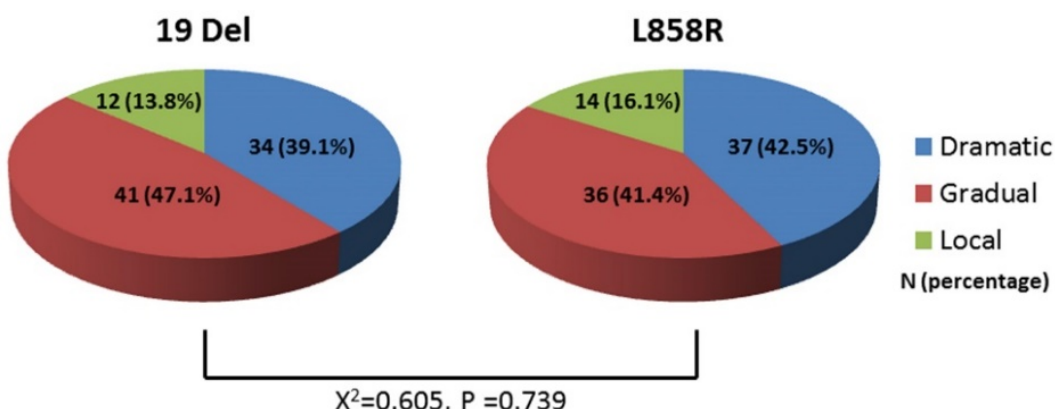

Figure 2. Comparison of the distribution of EGFR-TKI failure modes between 19 Del and L858R. Abbreviations:19 Del, exon 19 deletion; L858R, exon 21 L858R mutation. 

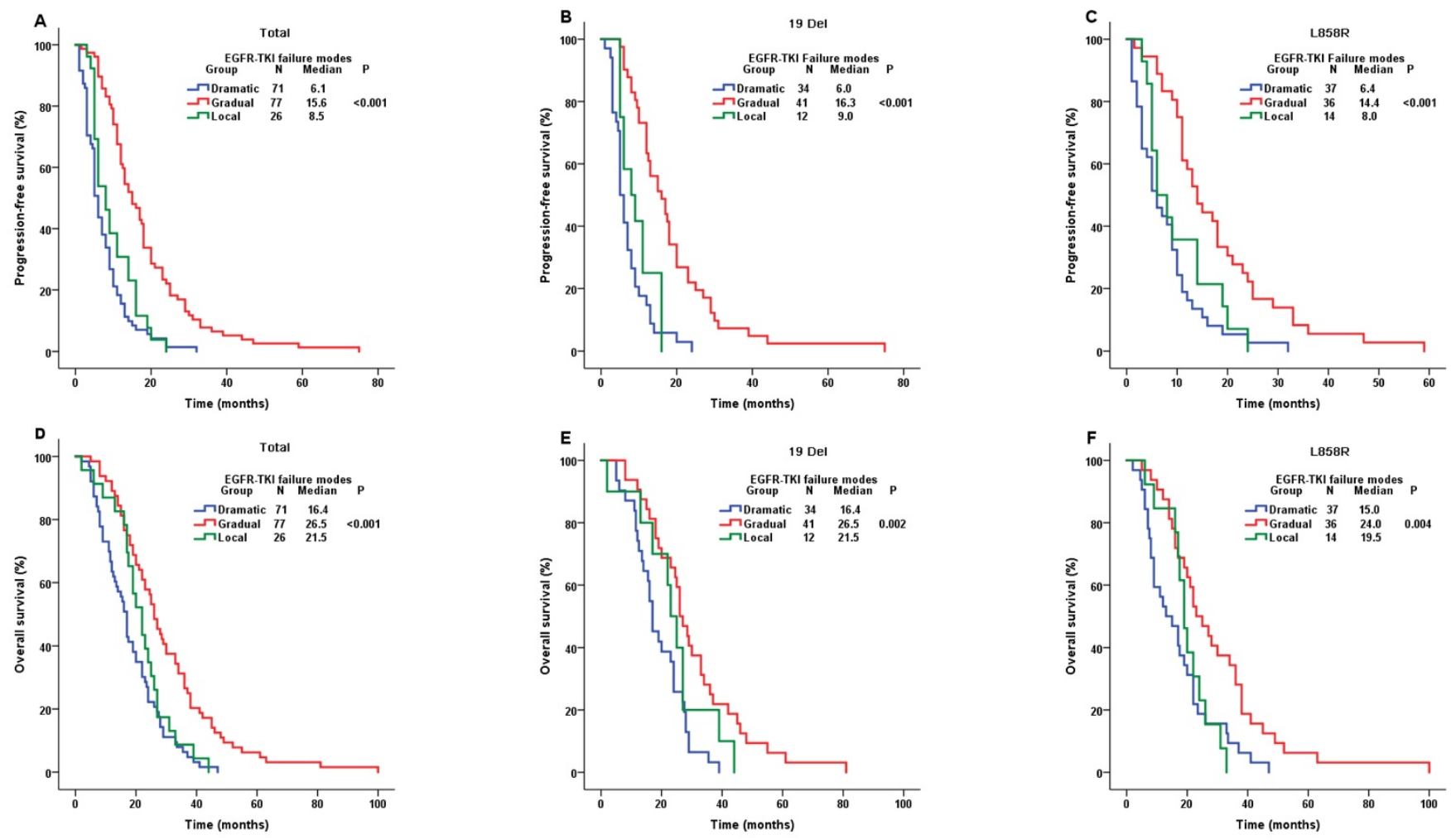

Figure 3. Comparison of PFS and OS among different EGFR-TKI failure modes. A. PFS in the whole population; B. PFS in the 19 Del cohort; C. PFS in the L858R cohort; D. OS in the whole population; E. OS in the 19 Del cohort; F. OS in the L858R cohort; Abbreviations:19 Del, exon 19 deletion; L858R, exon 21 L858R mutation; EGFR, epidermal growth factor receptor; TKI, tyrosine kinase inhibitor; PFS, progression-free survival; OS, overall survival.

\section{Discussion}

Yang's previous study showed different clinical modes of EGFR-TKI failure could favor corresponding strategies for subsequent treatment $[13,16]$. Developing strategy for subsequent treatment after EGFR-TKI failure according to T790M tests and patients' symptom burden had been adopted by National Comprehensive Cancer Network(NCCN)guideline for NSCLC version 3.2017. Continuing 1st- generation EGFR-TKI is recommended for asymptomatic progression and treatment should be various for symptomatic progression [17, 18]. Particularly, more and more evidences suggested that 19 Del and L858R are two distinct EGFR-positive diseases [5, 6, 7, 19]. Hence, we believe that it is necessary to take EGFR mutation subgroups into consideration for making choices of subsequent treatment after EGFR-TKI failure. Our study suggested that although the distribution of clinical EGFR-TKI failure modes was similar between 19 Del and L858R, different subsequent strategies should be given to those two diseases respectively to obtain survival benefit in each TKI failure mode.

Previous NCCN guideline suggested that patients suffered severe symptoms after acquired resistance to EGFR-TKI, which meant dramatic progression in another word, should turn to chemotherapy or osimertinib (if patients were T790M positive) $[17,18,20]$. Our study discovered that in 19 Del subgroup, patients switching to chemotherapy had longer OS than those with TKI continuation or BSC. On the contrary, in L858R subgroup, patients with previous TKI continuation demonstrated a numerical longer OS than those switching to chemotherapy or BSC. Thus, subsequent strategies should be different according to EGFR mutation status. For 19 Del patients, treatment according to the above NCCN guideline mentioned was suitable. However, for L858R patients, either continuing previous TKI or osimertinib (if T790M positive) would be appropriate.

Moreover, NCCN guideline suggested asymptomatic patients, that is gradual progression, may continue using previous TKI or turn to osimertinib (if T790M positive). The ASPIRATION study supported that erlotinib therapy in EGFR-positive NSCLC patients beyond progression was feasible [21]. Our study discovered that only L858R patients would obtain survival benefit from continuing previous TKI. However, we found that 19 Del patients could not get survival benefit from TKI continuation or chemotherapy. Therefore, we suggested, for gradual progression cases, 19 Del patients should turn to osimertinib (if T790M positive), while L858R patients should continue using previous TKI or turn to osimertinib (if T790M positive). 

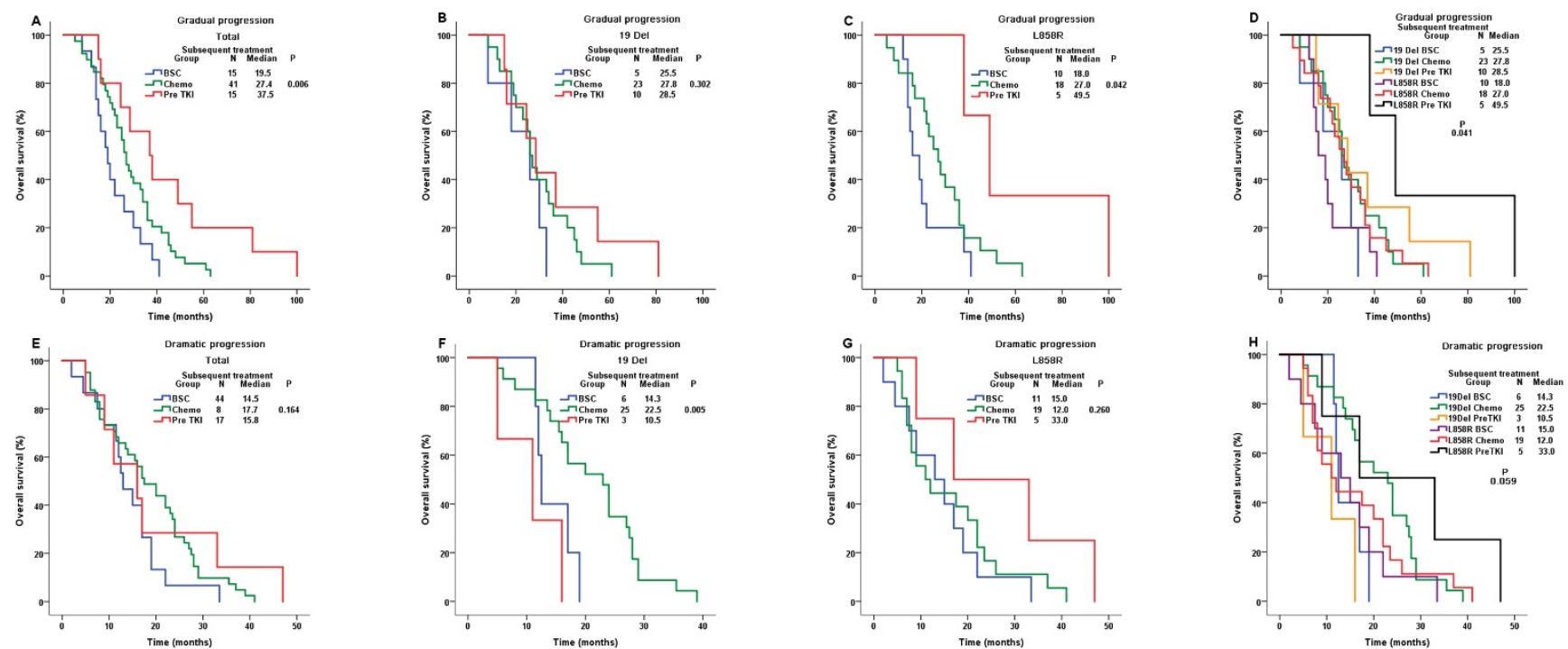

Figure 4. Comparison of OS among different subsequent treatment in gradual progression group and dramatic progression group. For gradual progression group: A. the whole population; B. the 19 Del cohort; C. the L858R cohort; D. the 19 Del \& the L858R cohort. For dramatic progression group: E. the whole population; F. the 19 Del cohort; G. the L858R cohort; $\mathrm{H}$. the 19 Del \& the L858R cohort; Abbreviations:19 Del, exon 19 deletion; L858R, exon 21 L858R mutation; BSC, best supportive care; Chemo, chemotherapy; Pre TKI, previous 1st-generation tyrosine kinase inhibitor; OS, overall survival.

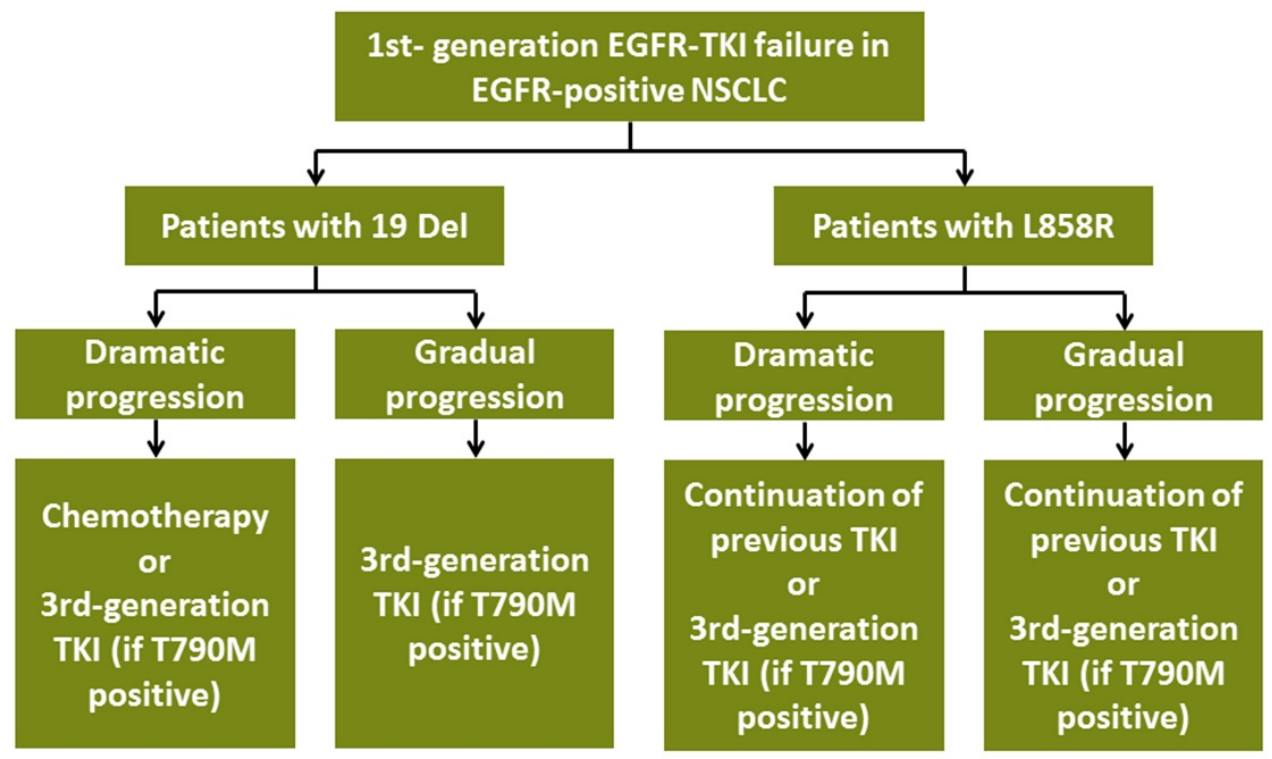

Figure 5. Subsequent treatment for advanced EGFR-positive NSCLC patients with acquired resistance to TKI according to EGFR mutation subtypes. Abbreviations: 19 Del, exon 19 deletion; L858R, exon 21 L858R mutation; T790M, exon 20 T790M mutation; EGFR, epidermal growth factor receptor; TKI, tyrosine kinase inhibitor; NSCLC, non-small-cell lung cancer

For local progression patients, NCCN guideline suggested that continuation of previous TKI/ switching to osimertinib (if T790M positive) plus local interventions such as radiotherapy were appropriate [22, 23, 24, 25]. Restricted by limited sample size, we could not conduct subgroup analyses stratified by 19 Del and L858R. Further studies are needed to explore proper management for $19 \mathrm{Del}$ and L858R patients with local progression.

Our study raised a novel treatment pattern of choosing different subsequent measure according to EGFR mutation subtypes after acquired EGFR-TKI failure (Figure 5). However, it still had several intrinsic limitations. Firstly, our study was retrospective design with limited sample size, which might cause relevant bias. Prospective studies are warranted to validate our results. Besides, most of patients with acquired EGFR-TKI failure were unwilling to re-biopsy, which led to unavailable drug resistant tumor samples. As a result, we could not conduct the comparisons of molecular backgrounds of EGFR-TKI resistance between 19 Del and L858R patients in the level of genomics, transcriptomics, proteomics and metabonomics in each clinical failure modes. Future studies are needed to explore the molecular modes TKI resistance between $19 \mathrm{Del}$ and 
L858R behind the phenomenon. In addition, we only focused on the common two types of EGFR mutation for analyses. Recent studies showed uncommon mutation like L861Q mutation, G719X (G719S/A/C) mutation, S768I mutation, exon 20 insertions might respond to EGFR-TKIs differently [26, 27]. Whether the subsequent strategies for these rare mutants should be different after TKI failure still remains unclear.

It is well known that $19 \mathrm{Del}$ and L858R are two distinct EGFR-positive diseases. However, the clinical significance of those two different diseases is questionable. Our work was the first study which found the clinical significance of taking 19 Del and L858R into different diseases. It might change the treatment pattern after TKI failure based on EGFR mutation subtypes stratified by 19 Del and L858R. Although the clinical TKI failure modes showed similar distributions between 19 Del and L858R, subsequent treatment should be different according to EGFR mutation subtypes after acquired EGFR-TKI failure. 19 Del patients with dramatic progression would obtain survival benefit from chemotherapy, while those with gradual progression got no survival benefit neither from chemotherapy nor previous TKI continuation. Moreover, for L858R patients, both those with dramatic and gradual progression would benefit from previous TKI continuation.

\section{Supplementary Material}

Supplementary tables.

http://www.jcancer.org/v08p1865s1.pdf

\section{Acknowledgement}

This work was supported by National Key R\&D Program of China 2016YFC0905500.

\section{Competing Interests} interest.

The authors have declared no conflicts of

\section{References}

1. Maemondo $M$, Inoue $A$, Kobayashi $K$ et al. Gefitinib or chemotherapy for non-small-cell lung cancer with mutated EGFR. $N$ EnglJ Med 2010;362:2380-2388.

2. Rosell R, Carcereny E, Gervais R et al. Erlotinib versus standard chemotherapy as first-line treatment for European patients with advanced EGFR mutation-positive non-small-cell lung cancer (EURTAC): a multicentre, open-label, randomised phase 3 trial. Lancet Oncol 2012; 13: 239-246.

3. Mok T, Yang JJ, Lam KC. Treating patients with EGFR-sensitizing mutations: first line or second line--is there a difference? J ClinOncol 2013; 31: 1081-1088.

4. Wu YL, Zhou C, Hu CP et al. Afatinib versus cisplatin plus gemcitabine for first-line treatment of Asian patients with advanced non-small-cell lung cancer harbouring EGFR mutations (LUX-Lung 6): an open-label, randomised phase 3 trial. Lancet Oncol 2014; 15: 213-222.

5. Jackman DM, Yeap BY, Sequist LV et al. Exon 19 deletion mutations of epidermal growth factor receptor are associated with prolonged survival in non-small cell lung cancer patients treated with gefitinib or erlotinib. Clin Cancer Res 2006; 12: 3908-3914.

6. Yang JC, $\mathrm{Wu} \mathrm{YL}$, Schuler $\mathrm{M}$ et al. Afatinib versus cisplatin-based chemotherapy for EGFR mutation-positive lung adenocarcinoma (LUX-Lung
3 and LUX-Lung 6): analysis of overall survival data from two randomised, phase 3 trials. Lancet Oncol 2015; 16: 141-151.

7. Zhang Y, Sheng J, Kang S et al. Patients with exon 19 deletion were associated with longer progression-free survival compared to those with L858R mutation after first-line EGFR-TKIs for advanced non-small cell lung cancer: a meta-analysis. PLoS One 2014; 9: e107161.

8. Zhang $Y, H e$ D, Fang $W$ et al. The Difference of Clinical Characteristics Between Patients With Exon 19 Deletion and Those With L858R Mutation in Nonsmall Cell Lung Cancer. Medicine 2015; 94: e1949.

9. Shih JY, Gow $\mathrm{CH}$, Yang PC. EGFR mutation conferring primary resistance to gefitinib in non-small-cell lung cancer. N Engl J Med 2005; 353: 207-208.

10. Pao W, Miller VA, Politi KA et al. Acquired resistance of lung adenocarcinomas to gefitinib or erlotinib is associated with a second mutation in the EGFR kinase domain. PLoS Med 2005; 2: e73.

11. Kosaka T, Yatabe Y, Endoh $\mathrm{H}$ et al. Analysis of epidermal growth factor receptor gene mutation in patients with non-small cell lung cancer and acquired resistance to gefitinib. Clin Cancer Res 2006; 12: 5764-5769.

12. Engelman JA, Zejnullahu K, Mitsudomi $\mathrm{T}$ et al. MET amplification leads to gefitinib resistance in lung cancer by activating ERBB3 signaling. Science 2007; 316: 1039-1043.

13. Yang JJ, Chen HJ, Yan $\mathrm{HH}$ et al. Clinical modes of EGFR tyrosine kinase inhibitor failure and subsequent management in advanced non-small cell lung cancer. Lung Cancer 2013; 79: 33-39.

14. Eisenhauer EA, Therasse P, Bogaerts J et al. New response evaluation criteria in solid tumours: revised RECIST guideline (version 1.1). Eur J Cancer 2009; 45: 228-247.

15. vanPuijenbroek $\mathrm{R}$, Bosquée $\mathrm{L}$, Meert $\mathrm{AP}$ et al. Gefitinibmonotherapy in advanced nonsmall cell lung cancer: a large Western community implementation study. EurRespir J 2007; 29: 128-133.

16. Gandara DR, Li T, Lara PN et al. Acquired resistance to targeted therapies against oncogene-driven non-small-cell lung cancer: approach to subtyping progressive disease and clinical implications. Clin Lung Cancer 2014; 15: 1-6.

17. Katakami N, Atagi S, Goto K et al. LUX-Lung 4: a phase II trial of afatinib in patients with advanced non-small-cell lung cancer who progressed during prior treatment with erlotinib, gefitinib, or both. J ClinOncol 2013; 31: 3335-3341.

18. Hirsh V, Cadranel J, Cong XJ et al. Symptom and quality of life benefit of afatinib in advanced non-small-cell lung cancer patients previously treated with erlotinib or gefitinib: results of a randomized phase $\mathrm{IIb} / \mathrm{III}$ trial (LUX-Lung 1). J Thorac Oncol 2013; 8: 229- 237.

19. Wang $\mathrm{Y}, \mathrm{Li} \mathrm{RQ}, \mathrm{Ai} \mathrm{YQ}$ et al. Exon 19 deletion was associated with better survival outcomes in advanced lung adenocarcinoma with mutant EGFR treated with EGFR-TKIs as second-line therapy after first-line chemotherapy: a retrospective analysis of 128 patients. Clin Transl Oncol 2015; 17: 727-736.

20. Miller VA, Hirsh V, Cadranel J et al. Afatinib versus placebo for patients with advanced, metastatic non-small-cell lung cancer after failure of erlotinib, gefitinib, or both, and one or two lines of chemotherapy (LUX-Lung 1): a phase 2b/3 randomised trial. Lancet Oncol 2012; 13: 528- 538.

21. Park K, Yu CJ, Kim SW et al. First-Line Erlotinib Therapy Until and Beyond Response Evaluation Criteria in Solid Tumors Progression in Asian Patients With Epidermal Growth Factor Receptor Mutation-Positive Non-Small-Cell Lung Cancer: The ASPIRATION Study. JAMA Oncol 2016; 2: 305-312.

22. Iyengar $\mathrm{P}$, Kavanagh $\mathrm{BD}$, Wardak $\mathrm{Z}$ et al. Phase II trial of stereotactic body radiation therapy combined with erlotinib for patients with limited but progressive metastatic non-small-cell lung cancer. J Clin Oncol 2014; 32: 3824-3830.

23. Simone CB 2nd, Burri SH, Heinzerling JH. Novel radiotherapy approaches for lung cancer: combining radiation therapy with targeted and immunotherapies. Transl Lung Cancer Res 2015; 4: 545-552.

24. Campo M, Al-Halabi H, Khandekar M et al. Integration of Stereotactic Body Radiation Therapy With Tyrosine Kinase Inhibitors in Stage IV Oncogene-Driven Lung Cancer. Oncologist 2016; 21: 964-973.

25. Becker $\mathrm{K}, \mathrm{Xu} \mathrm{Y}$. Management of tyrosine kinase inhibitor resistance in lung cancer with EGFR mutation. World J ClinOncol 2014; 5: 560-567.

26. $\mathrm{Xu} \mathrm{J}$, Jin $\mathrm{B}, \mathrm{Chu} \mathrm{T}$ et al. EGFR tyrosine kinase inhibitor (TKI) in patients with advanced non-small cell lung cancer (NSCLC) harboring uncommon EGFR mutations: A real-world study in China. Lung Cancer 2016; 96: 87-92.

27. Baek JH, Sun JM, Min YJ et al. Efficacy of EGFR tyrosine kinase inhibitors in patients with EGFR-mutated non-small cell lung cancer except both exon 19 deletion and exon 21 L858R: a retrospective analysis in Korea. Lung Cancer 2015; 87: 148-154. 